\title{
Comparison of gliding box and box-counting methods in river network analysis
}

\author{
A. Saa ${ }^{1}$, G. Gascó ${ }^{1}$, J. B. Grau ${ }^{2}$, J. M. Antón ${ }^{2}$, and A. M. Tarquis ${ }^{2}$ \\ ${ }^{1}$ Dpto. de Edafología y Climatología, E.T.S. de Ingenieros Agrónomos, Universidad Politécnica de Madrid. Ciudad \\ Universitaria s.n., 28040 Madrid, Spain \\ ${ }^{2}$ Dpto. de Matemática Aplicada a la Ingeniería Agronómica, E.T.S. de Ingenieros Agrónomos, Universidad Politécnica de \\ Madrid, Ciudad Universitaria s.n., 28040 Madrid, Spain
}

Received: 10 July 2007 - Revised: 23 August 2007 - Accepted: 3 September 2007 - Published: 12 September 2007

\begin{abstract}
We use multifractal analysis to estimate the Rényi dimensions of river basins by two different partition methods. These methods differ in the way that the Euclidian plane support of the measure is covered, partitioning it by using mutually exclusive boxes or by gliding a box over the plane.

Images of two different drainage basins, for the Ebro and Tajo rivers, located in Spain, were digitalized with a resolution of $0.5 \mathrm{~km}$, giving image sizes of $617 \times 1059$ pixels and $515 \times 1059$, respectively. Box sizes were chosen as powers of 2 , ranging from $2 \times 4$ pixels to $512 \times 1024$ pixels located within the image, with the purpose of covering the entire network. The resulting measures were plotted versus the logarithmic value of the box area instead of the box size length.

Multifractal Analysis (MFA) using a box counting algorithm was carried out according to the method of moments ranging from $-5<\mathrm{q}<5$, and the Rényi dimensions were calculated from the $\log / \log$ slope of the probability distribution for the respective moments over the box area. An optimal interval of box sizes was determined by estimating the characteristic length of the river networks and by taking the next higher power of 2 as the smallest box size. The optimized box size for both river networks ranges from $64 \times 128$ to $512 \times 1024$ pixels and illustrates the multiscaling behaviour of the Ebro and Tajo. By restricting the multifractal analysis to the box size range, good generalized dimension $\left(D_{q}\right)$ spectra were obtained but with very few points and with a low number of boxes for each size due to image size restrictions. The gliding box method was applied to the same box size range, providing more consistent and representative $D_{q}$ values. The numerical differences between the results, as well as the standard error values, are discussed.
\end{abstract}

Correspondence to: A. M. Tarquis

(anamaria.tarquis@upm.es)

\section{Introduction}

The analysis of the quantitative description of river networks has a long history. Already more than half a century ago, Horton (1932 and 1945) first studied the origin of river networks and the laws governing their dendritic structures, proposing a hierarchical classification of the streams belonging to the river that were later used by several authors (Mesa and Gupta, 1987). Years later, based on the contributions made by Mandelbrot (1977, 1982), several authors (Hjelmfelt, 1988; La Barbera and Rosso, 1989; Tarboton et al., 1990; Veltri et al., 1996, among others) applied a fractal analysis to the river network. They related the fractal dimension estimated to the bifurcation ratio, length ratio and area ratio defined in the hortonian laws. For a while a big controversy was created about the plane-filling nature of the network rivers, until it was concluded that these sets exhibit a self-affine fractal behavior (Ijjasz-Vasquez et al., 1992; Peckham, 1995; Claps and Olivetto, 1996; Maritan et al., 1996; Beauvais and Montgomery, 1997).

Fractal and multifractal concepts have been increasingly applied in various fields of science for describing complexity and self-similarity in nature, e.g. Rinaldo et al. (1993) and Rodriguez-Iturbe and Rinaldo (1997) considered the river networks as self-organized spatial structures. A geometrical multifractal (Tel and Vicsek, 1987; Vicsek et al., 1990) is a nonuniform fractal which unlike a uniform fractal exhibits local density fluctuations. Its characterization requires not a single dimension but a sequence of generalized fractal dimensions $\left(D_{q}\right)$. A multifractal analysis (MFA) to extract these dimensions from a network river may have utility, if there is marked variation in local density. In more general terms, multifractal formalism involves decomposing selfsimilar measures into intertwined fractal sets, which are each characterized by their singularity strength and fractal dimension. This type of analysis was first applied to the river network by Ijjasz-Vasquez et al. (1992) and Rinaldo et al. (1992)

Published by Copernicus Publications on behalf of the European Geosciences Union and the American Geophysical Union. 
to study the multifractal or multiscaling nature (Coniglio and Zannetti, 1989). Beside river network geometry (De Bartolo et al., 2000), other important hydrological variables, such as catchments areas, slopes (Rodriguez-Iturbe et al., 1994), topography (Lavallée et al., 1993; Veneziano and Niemann, 2000a, b), network optimization (Rigon et al., 1993), Rinaldo and Rodriguez-Iturbe, 1998), dissipation energy (Rinaldo et al., 1991; Ijjász-Vasquez et al., 1993), channel initiation function and the width function (Rodriguez-Iturbe and Rinaldo, 1997) have been studied through MFA.

Recent studies showed that the fractal dimensions can be used to model the hydrologic response of a river basin through the Fractal Instantaneous Unit Hydrograph (FIUH) (Fiorentino et al., 2002; Cudennec et al., 2004). This concept was extended to the multifractal analysis, through the use of the singularity spectrum, $f(\alpha)$, by Gaudio et al. (2006), who proposed the Multifractal Instantaneous Unit Hydrograph (MIUH). Therefore, in the studies of the hydrologic response in geomorphological terms, the numerical assessment of the generalized fractal dimensions which appear as exponents in the probability distribution function of travel times is of great importance (Rinaldo et al., 1991), and several authors are trying to related it to flood peaks (Gupta et al., 1994; S. G. de Bartolo, personal communication).

Reviewing the literature reveals that obtaining a reliable value of the generalized dimensions is not an easy calculation (Tel et al., 1989). Vicsek (1990) proposed practical methods to compute the $D_{q}$. He assumed the box size to be much greater than the smallest elementary cell in the system and much smaller than the system size. Merits and limitations of the MFA have been discussed by Chhabra et al. (1989), Aharony (1989, 1990), Beghdadi et al. (1993), Andraud et al. (1994), Meakin (1998), Bird et al. (2006), Dathe et al. (2006) and Perrier et al. (2006).

The difficulties arising, in practice, are due to the fact that the relevant quantities used in the multifractal concept are estimated asymptotically, and in this case, these estimations are coarse and limited by the finite resolution of the image (Ahammer et al., 2003) and the measure build on it, as the number of pixels representing the object in a box (Buczhowski et al., 1998). In other words, the theoretical limit (when the box-size tends to zero) cannot be reached in practice. On the other hand, MFA normally involves partitioning the space of study into nonoverlapping boxes to construct samples with multiple scales, known as the box counting (BC) method. The number of samples at a given scale, applying BC, is restricted by the size of the partitioning space and data resolution, which is usually another main factor influencing statistical estimation in MFA (Cheng and Agterberg, 1996). To avoid these problems several methods have been proposed (Liebovitch and Toth, 1989; Block et al., 1990; Veneziano et al., 1995; Yamaguti and Prado, 1997; Oiwa and Fiedler-Ferrara, 1998; Feeny et al., 2000) and a few applied to the river network, such as the sand box method (De Bartolo et al., 2004), generalization of the correlation method
(De Bartolo et al., 2006; Gaudio et al., 2006), and the fixed mass method (De Bartolo et al., 2006).

The two objectives of this work are to study: 1) the effect of the range box sizes chosen when a BC method is applied, 2) the effect of the subdivision method applied in the calculation of generalized fractal dimensions $\left(D_{q}\right)$, comparing the BC method and the gliding box method (GB) that has been applied by Grau et al. (2006) in pore systems but never in the river networks. With these purposes two river basin images with distinctive spatial arrangement were analyzed to obtain their $D_{q}$ values.

\section{The concept of multifractals}

Multifractal analysis and the different representations that can be made are complex (see Mach et al., 1995, and references therein); we will try to summarize this in the case presented here. MFA in 2-D images involves partitioning the plane into boxes to construct samples with multiple scales. The BC method combines pixels to form larger mutually exclusive boxes, each containing a different set of pixels. In this case, to cover as maximum as area as possible, centered in the middle of the image, we begin with a rectangle of $L_{1} \times L_{2}$ pixels (an area $A$ ), and a partitioned process is applied with a box size $r_{1} \times r_{2}$, corresponding to an area of $a$, then the number of boxes with size $a(n(a))$ will follow the proportion:

$n(a) \propto\left(\frac{A}{a}\right)$.

Higher is the size of the boxes $(a)$ lower is the number of boxes $(n(a))$ and it might not be large enough to properly represent the distribution of the measure over the plane.

The GB method was originally used for lacunarity analysis (Allain and Cloitre, 1991) and later was modified by Cheng (1997a, b) to apply it to MFA and used by Grau et al. (2006) in pore systems. Basically, the GB method constructs samples by gliding a box of certain size $(a)$ over the grid map in all possible directions. An "up-scaling" partitioning process will begin with a minimum size or area box $\left(a_{\min }\right)$ up to a certain size less than $A$. If the minimum size box corresponds to $a_{\min }=r_{1} \min \times r_{2} \min$, then the proportionality of the number of boxes of size $a=r_{1} \times r_{2}\left(n^{*}(a)\right)$ is:

$n^{*}(a) \propto\left(\frac{L_{1}-r_{1}+r_{1} \min }{r_{1} \min }\right) \times\left(\frac{L_{2}-r_{2}+r_{2} \min }{r_{2} \min }\right)$.

In our case one length size is double the other one, $L_{2}=2 \times L_{1}$, and this proportion remains for all the parameters in Eq. (2) $\left(r_{2}, r_{2} \min \right)$. So we can conclude:

$n^{*}(a) \propto\left(\frac{L_{1}-r_{1}+r_{1 \min }}{r_{1 \min }}\right)^{2}$

being $a_{\min } \leq a \leq A$. 
An advantage of the GB method is the large sample size that usually leads to better statistical results. Because this partitioning overlaps, the measure defined on these boxes is not statistically independent and the definition of the measure in the gliding boxes is different, as explained below.

\subsection{Box-counting method}

Generalized dimensions calculated using the $\mathrm{BC}$ technique basically accounts for the mass contained in each box. An image is divided into $n(a)$ boxes, and for each box the fraction of the river network in that box is calculated $\left(\mu_{i}\right)$

$$
\mu_{i}=\frac{m_{i}}{m_{T}}=\frac{m_{i}}{\sum_{i=1}^{n(a)} m_{i}},
$$

where $m_{i}$ is the number of river network pixels (for example, black pixels) and $m_{T}$ is the total number of black pixels in an image. In this case, the river network space area is the measure, whereas the support is the rectangle grid and $\mu_{i}$ can be interpreted as a probability measure (Plotnick et al., 1996). In practice, using the box counting method, for every box $i$ the probability of "containing object", also called the partition function $(\chi(q, a))$, is obtained for different moments $q$, which can vary from $-\infty$ to $+\infty$.

$\chi(q, a)=\sum_{i=1}^{n(a)}\left[\chi_{i}(q, a)\right] \quad q \in \Re$

where $\chi_{i}(q, a)=\mu_{i}^{q} . \quad \chi_{i}$ is a weighted measure that represents the percentage of black pixels in the $i$ th box, and $q$ is the weight or moment of the measure. When computing boxes of size $a$, the possible values of $m_{i}$ are from 0 to $a$. So let $N_{j}(a)$ be the number of boxes containing $j$ pixels of network space in that grid. Equation (5) will then be (Barnsley et al., 1988):

$\chi(q, a)=\sum_{i=1}^{n(a)}\left(\mu_{i}\right)^{q}=\sum_{i=1}^{n(a)}\left(\frac{m_{i}}{m_{T}}\right)^{q}=\sum_{j=1}^{a} N_{j}(a)\left(\frac{j}{m_{T}}\right)^{q}$

being $m_{T}=\sum_{j=1}^{a} j N_{j}(a)$.

Using the distribution function the $N_{j}(a)$ calculation becomes simpler and computational errors are smaller (Barnsley et al., 1988). This has been extensively used in black and white images (see Tarquis et al., 2003, and references therein).

A log-log plot of a self-similar measure, $\chi(q, a)$, vs. $a$ at various values for $q$ gives:

$\chi(q, a) \sim a^{\tau(q)}$,

where $\tau(q)$ is the $q$ th mass exponent (Hentschel and Procaccia, 1983; Feder, 1989). We can express $\tau(q)$ as:

$\tau(q)=\lim _{a \rightarrow 0} \frac{\log (\chi(q, a))}{\log (a)}$.
Based on the work of Rényi (1955) the generalized dimension, $D_{q}$, can be introduced by the following scaling relationship (Grassberger, 1983; Hentschel and Procaccia, 1983; Hasley et al., 1986; Feder, 1989):

$D_{q}=\lim _{r \rightarrow 0} \frac{\log [\chi(q, a)]}{(q-1) \log a}$.

The sum in the numerator of Eq. (9) is dominated by the highest values of $\mu_{i}$ for $q>0$, and by the lowest values of $\mu_{i}$ for $q<0$; therefore,

$\tau(q)=(q-1) D_{q} \quad$ or $\quad D_{q}=\frac{\tau(q)}{(q-1)}$.

Moments for $q=1,2 \ldots$ describe mathematically defined fractal dimensions. They can be found by inserting a $q$ value in Eqs. (9) or (10). For $q=1$ the calculation is trickier, Eq. (9) cannot be applied, and in this case we use the relationship:

$$
\begin{aligned}
D_{1} & =\lim _{a \rightarrow 0} \frac{\sum_{i=1}^{n(a)} \chi_{i}(1, a) \log \left[\chi_{i}(1, a)\right]}{\log a} \\
& =\lim _{a \rightarrow 0} \frac{\sum_{i=1}^{n(a)} \mu_{i}(a) \log \left[\mu_{i}(a)\right],}{\log a}
\end{aligned}
$$

which is the (classical) information dimension: the numerator in Eq. (11) corresponds to the entropy of a probability distribution. This is one of the analogies between multifractals and statistical mechanics. For $q=2$ the correlation dimension $D_{2}$ can be obtained as

$D_{2}=\lim _{a \rightarrow 0} \frac{\log \sum_{i=1}^{n(a)} \mu_{i}^{2}}{\log a}$.

It describes the probability of finding pixels belonging to the object within a given distance when starting on a pixel belonging to the object. Fractal dimensions for higher moments can be estimated, according to Eq. (9). Homogeneous structure is indicated when the $D_{q}$ values are close together, such as in a monofractal case, regardless which value has $q$.

\subsection{Gliding box method}

Similarly to the BC method, it is necessary to define the measure in the gliding boxes. Let $n^{*}(m, a)$ be the number of gliding boxes of size $a$ and mass (number of pore pixels) $m$. Dividing it by $n^{*}(a)$ the probability function $\beta(m, a)$ is obtained for a gliding box of size $a$ containing mass $m$.

The statistical moments of this distribution are:

$$
\begin{aligned}
\chi^{*}(q, a) & =\sum_{m=1}^{a} \beta(m, a) m^{q} \\
& =\frac{1}{n^{*}(a)} \sum_{i=1}^{n^{*}(a)} m_{i}^{q}(a) \approx E\left\{m^{q}\right\},
\end{aligned}
$$


where $\chi^{*}(q, a)$ is the $q$ th order moment of $\beta(m, a)$ and the sum is for all the gliding boxes with $m_{i}>0$.

The difference between $\chi^{*}(q, a)$ Eq. (13) and $\chi(q, a)$ is that:

$\chi(q, a) \approx\left(\frac{A}{a}\right) E\left\{m^{q}\right\}$.

Combining Eqs. (13) and (14) gives:

$\chi(q, a) \approx\left(\frac{A}{a}\right) \chi^{*}(q, a)$.

If we use this relation in Eqs. (8) and (9) we have that:

$$
\tau(q)=\lim _{r \rightarrow 0} \frac{\log \left(\chi^{*}(q, a)\right)}{\log (a)}+\lim _{a \rightarrow 0} \frac{\log \left(\frac{A}{a}\right)}{\log (a)} .
$$

The second limit is easy to resolve given a value of -1 . It is concluded that (Cheng, 1997a, b):

$\tau(q)=\lim _{a \rightarrow 0} \frac{\log \left(\chi^{*}(q, a)\right)}{\log (a)}-1$.

Once this estimation is done, Eq. (10) can be applied to estimate $D_{q}$. For the case of $q=1$ the following relationship is applied based on Cheng and Agerberg's (1996) work:

$D_{1}=\lim _{a \rightarrow 0} \frac{\frac{1}{n^{*}(a)} \sum_{i=1}^{n^{*}(a)} m_{i}(a) \times \log \left[m_{i}(a)\right]}{\log a}$.

\section{Material and methods}

\subsection{River networks}

The Ebro basin is located in the north of Spain and includes $85399 \mathrm{~km}^{2}$, from which 84452 are in Spain, $552 \mathrm{~km}^{2}$ in France and $445 \mathrm{~km}^{2}$ in Andorra. The Tajo basin includes $58941 \mathrm{~km}^{2}, 55645 \mathrm{~km}^{2}$ in Spain and $3296 \mathrm{~km}^{2}$ in Portugal. Both are large basins within the temperate zone of the world with water and sediment regimes controlled by climate (Sundborg and Jansson, 1991). These Spanish rivers have rain as the principal supply of water. Both rivers have a pluvial type of hydrological regime (Pardé, 1955), which in the Northern Hemisphere is characterized by a water discharge maximum during the winter season, when maximum precipitation combines with minimum evaporation. Minimum discharge occurs during summer, which is the warmest season. During the 1940-2006 period, the mean annual water flow of the Ebro river was $18.23 \mathrm{~km}^{3}$ year ${ }^{-1}$, from 29.73 to $8.39 \mathrm{~km}^{3}$ year $^{-1}$ (Gascó et al., 2004). The mean annual water flow of the Tajo river was $4.32 \mathrm{~km}^{3}$ year $^{-1}$ (maximum 6.57 and minimum 1.91), but this water flow actually diminished because of the Tajo-Segura diversion, which was as much as $0.6 \mathrm{~km}^{3}$ year $^{-1}$.

Black and white images of both basins were donated by CEDEX (Ministerio de Fomento) at $0.5 \mathrm{~km}$ of resolution. The Ebro and Tajo river basins had an image size of
$617 \times 1059$ and $515 \times 1059$ pixels, respectively. Once these images where transformed into a matrix of 0 (white pixel) and 1 (black pixel) the mass distribution of black pixels was analyzed by applying a multifractal analysis and extracting the generalized fractal dimensions.

\subsection{Multifractal analysis}

MFA has been carried out using first a box counting algorithm which estimates the probability of containing mass (river network) for every single box of size $1 \times 2$ to $512 \times 1024$ pixels in steps of $(1 \times 2) \times 2^{k}, 0 \leq k \leq 9$. For both images a size of $512 \times 1024$ pixels was selected without losing data from the river network; in doing so, this avoids artifacts which can occur when the boxes do not cover the image entirely at the borders. The generalized dimensions are obtained as the slope of the partition function over box size, both taken as logarithm (Eq. 6b) and the relation $\tau(q)=(1-q) D_{q}$. This method is known as the method of moments (Evertsz and Mandelbrot, 1992), as $D_{q}$ is estimated for every moment $q$. $D_{1}$ is simply determined as $\tau$ (Eq. 1 ).

The computational implementation to calculate the partition function is taking into account that we have a binary image where each pixel is either 0 or 1 ; see Tarquis et al. (2003) for more details. A crucial step in MFA is to determine the range of box sizes and the range of order $q$ over which this analysis is applied (Saucier and Muller, 1998).

The minimum box size $\left(a_{\min }\right)$ is obtained here based on configuration entropy analysis, which is easy to implement and has already been used for several types of images (Tarquis et al., 2006; Dathe et al., 2006). Configuration entropy analysis studies the effect of scale in any measure (a scalar quantity that leads to a positive distribution) defined in a plane. A distribution of the measure is obtained when the measure is estimated for different sizes of areas and every sub-area (Andraud et al., 1994). For small sub-areas (or boxes in an image), the local distributions show a certain behavior that can characterize the spatial pattern of the larger image.

In the work presented, the configuration entropy is estimated for the river network. The distribution of local porosity in a rectangular lattice image of size $L_{1} \times L_{2}$ is obtained by subdividing the image into $n(a)$ boxes of size $a$ from $a=1 \times 2$ to $a=\frac{1}{8}\left(L_{1} \times L_{2}\right)$, and in every box the number of pixels belonging to the river network is recorded.

The probability of $j$ pixels representing the network in a box of size $a\left(p_{j}(a)\right)$ is defined as:

$p_{j}(a)=\frac{N_{j}(a)}{n(a)}$,

where $N_{j}(a)$ is the number of boxes with $j$ pixels representing the network river and $n(a)$ is the number of boxes of area $a$. The configuration entropy $H$ (Andraud et al., 1997)

$H(a)=-\sum_{j=0}^{a} p_{j}(a) \log \left(p_{j}(a)\right)$ 


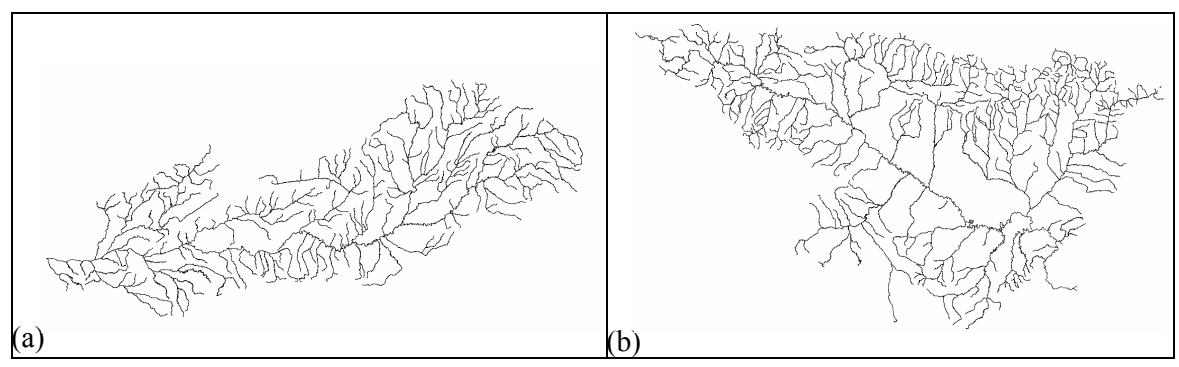

Fig. 1. River network binary images at $0.5 \mathrm{~km}$ resolution: (a) Tajo and (b) Ebro.

Table 1. Characteristic length obtained for the river network from the respective binary images, using the configuration entropy method. The minimum box area chosen for the multifractal analysis is the next higher value $2^{k}$ that multiplies the elementary box $2 \times 4$ pixels.

\begin{tabular}{ccccc}
\hline River & $\begin{array}{c}\text { Characteristic area } \\
\left(a_{\min }\right)\end{array}$ & $\begin{array}{c}\text { Maximum entropy } \\
\left(\mathrm{H}^{*}\left(a_{\min }\right)\right)\end{array}$ & $\begin{array}{c}\text { Minimum area size } \\
\text { pixels }\end{array}$ & $\begin{array}{c}\text { Maximum area size } \\
\text { pixels }\end{array}$ \\
\hline Tajo & $53 \times 106$ & 0.848 & $64 \times 128$ & $512 \times 1024$ \\
Ebro & $60 \times 120$ & 0.781 & $64 \times 128$ & $512 \times 1024$ \\
\hline
\end{tabular}

expresses the uncertainty of computing the network when covering an image with boxes of area $a$.

To compare entropy values corresponding to different box areas $a, H(a)$ is normalized to the normalized configuration entropy $H^{*}(a)$ :

$H^{*}(a)=\frac{H(a)}{H_{\max }(a)}$

with $H_{\max }(a)=\log (a+1)$.

The curve obtained when plotting the normalized entropy versus $a$ can be used as a descriptor of the image morphology. In many instances, the curve presents a local maximum $H^{*}\left(a_{\min }\right)$. The value where the maximum is located is called the characteristic length $a_{\min }$. The existence or non-existence of $a_{\text {min }}$ provides complementary information of spatial arrangements (Rodriguez-Iturbe and D'Odorico, 1998). Results with the characteristic length and the maximum entropy of the Tajo and Ebro rivers network investigated can be found in Table 1.

Once $D_{q}$ values have been extracted from both river networks, applying the BC method in the optimize box size range selected, the GB method was then applied. In this work we have applied the multiplier method to estimate $\tau(q)$ (see Cheng, 1999, for more details), which is based on the following equation:

$$
<\tau(q)>=-\frac{\log (<M(q, a)>)}{\log \left(\frac{a}{a_{\min }}\right)}-1,
$$

where $<>$ stands for the statistical moment, $M$ represents the multiplier measured on each pixel as:

$M(q, a)=\left(\frac{\mu\left(a_{\min }\right)}{\mu(a)}\right)^{q}$.
The advantage of using Eq. (22) in comparison with Eq. (17) is that the estimation is independent of box area $a$ which allows for the use of successive box sizes to estimate $\tau(q)$. Equation (23) imposes that $\mu\left(a_{\min }\right)$ shouldn't be null; otherwise the proportion $\left(\frac{\mu\left(a_{\min }\right)}{\mu(a)}\right)$ will always be 0 or undefined for any $a$ value.

Once $\tau(q)$ is estimated, Eq. (10) is applied to obtain the generalized dimensions.

\section{Results and discussion}

\subsection{The partition function}

Values for the partition function $\chi(q, a)$ have been estimated for the whole available box size range of $2 \times 4$ to $512 \times 1024$ pixels, in steps of $2^{k}, 0 \leq k \leq 8$. The partition functions reveal similar shapes for both river basins (Fig. 2). For $q>0$, the partition functions show a positive slope with a distinct linear behavior. For $q \leq 0$ the partition functions have a negative slope with a pronounced deviation from linearity for $q$ equal or close to -5 . The area size at which the behavior is different for the three images is around $64 \times 128$ pixels. These two phases were not evident with positive $q$ values (see Fig. 2).

The existence of a plateau phase of $\log (\chi(q, a))$ can be explained by the nature of the measure we were studying. At $a$ values close to $1 \times 2$, the variation in number of black pixels is based on a few pixels, having the most simplicity when $a=2$, where the measure can only have a value of 0 , 1 or 2 . Thus, for small areas the proportions among their values are mainly constant. However, when the area passes a certain size a scaling pattern begins. 


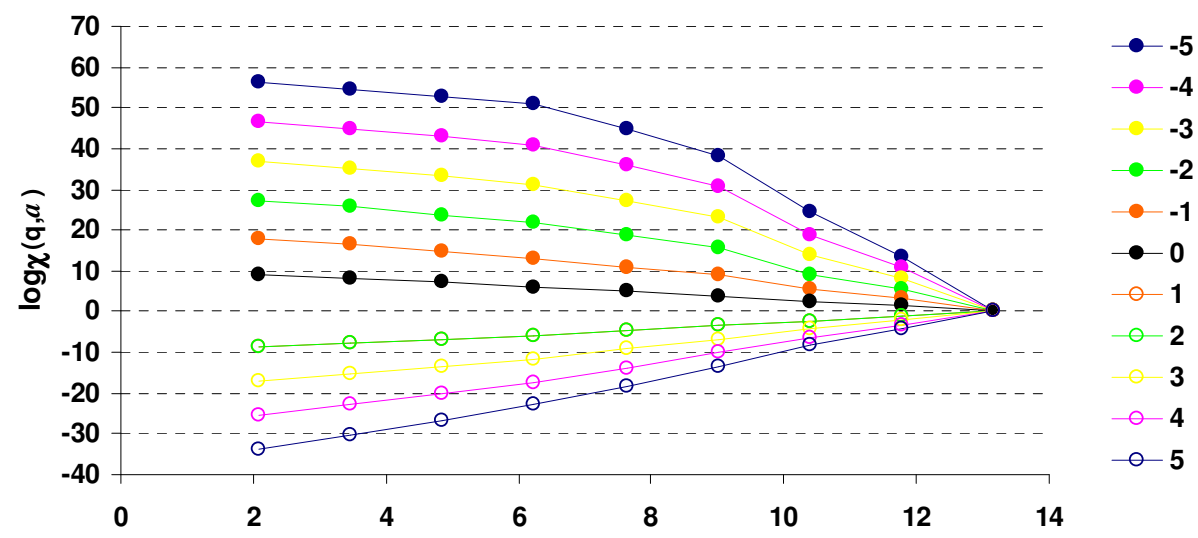

(a)

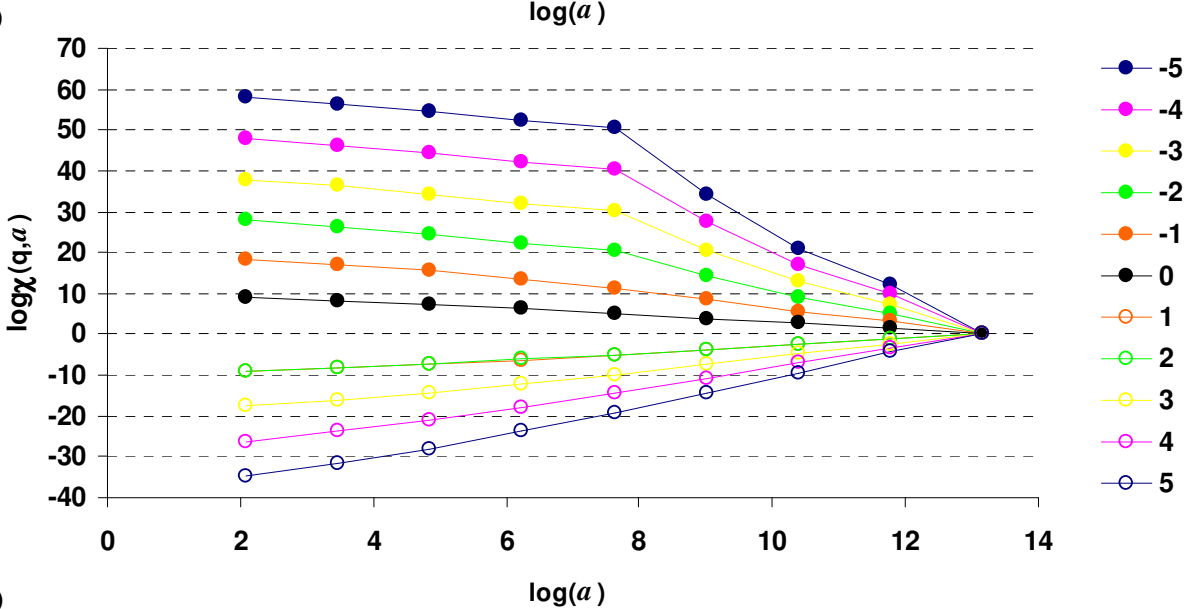

Fig. 2. Bi-log plot of $\chi(a, q)$ versus box area (a) at different mass exponent (q): (a) Tajo and (b) Ebro.

This involves coefficients of determination which are clearly smaller than 1 when estimating the mass exponent $\tau(q)$, according to Eq. (8).

\subsection{Generalized dimensions using the box counting method}

If we considered all of the regression points, the $D_{q}$ values obtained, mainly for $q<0$, were quite different from ones obtained when we restrict the range of areas to calculate the slope of the regression line (Fig. 3). Between all the possible criteria, any $D_{q}$ can be obtained, but for $q \geq 0$ the differences were not significant. Many authors have pointed out this fact since the first applications of multifractal analysis to experimental results (Vicsek, 1990; Evertsz and Mandelbrot, 1992; Crawford et al., 1999; Cheng, 1999; Bird et al., 2006; Tarquis et al., 2006; Grau et al., 2006).
An explanation as to why some coefficients of determination $\left(R^{2}\right)$ are rather poor can be found in the graphs of the partition function. When the slope of $\log (\chi(a, q))$ over $\log$ (a) does not yield a straight line for all $a$, different generalized dimensions will be obtained for specific ranges of $a$. This is why the range of box sizes chosen to determine $D_{q}$ is crucial regarding the final result, especially for negative $q$ moments.

Digital images (basically a matrix with 0 and 1 elements) always have a limited resolution, and only a few pixels can cause great changes in the partition function, especially for negative $q$. Following Evertsz and Mandelbrot (1992), it should be checked carefully whether the slope of $\chi(a, q)$ over $a$ (the mass exponent $\tau(q)$ ) yields a straight line before further data processing takes place.

The implications that $D_{q}$ changes so dramatically in this case make any comparison of the amplitude $\left(D_{-5}-D_{+5}\right) \mathrm{im}-$ possible, as it has been used in several works (Tarquis et al., 2001). The differences found among the $D_{q}$ representation are mainly found in the negative part.

The characteristic length obtained from configuration entropy analysis was taken as a measure for the smallest box 


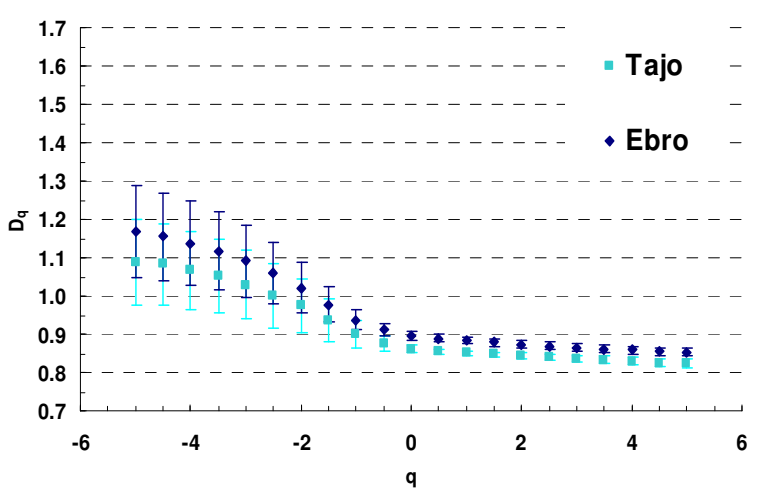

(a)

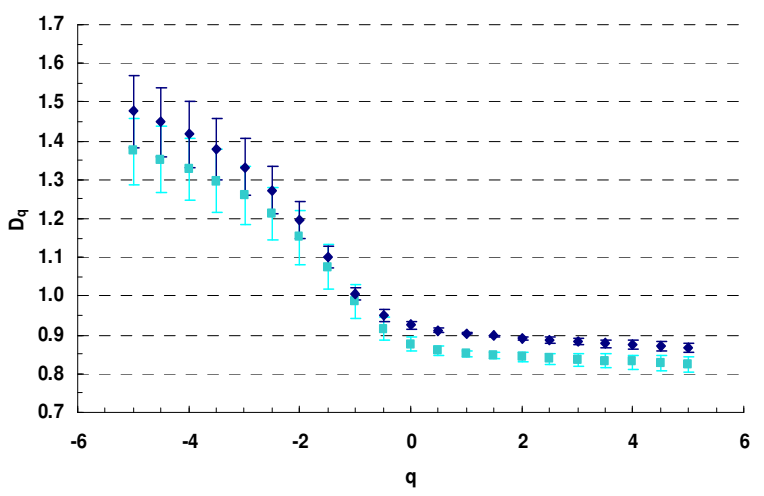

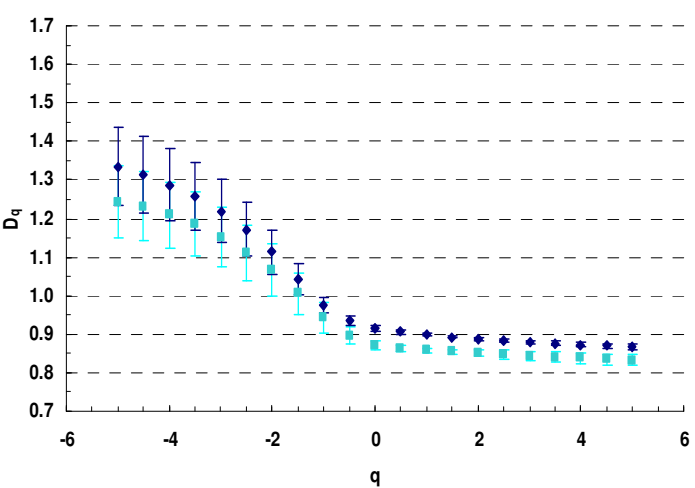

(b)

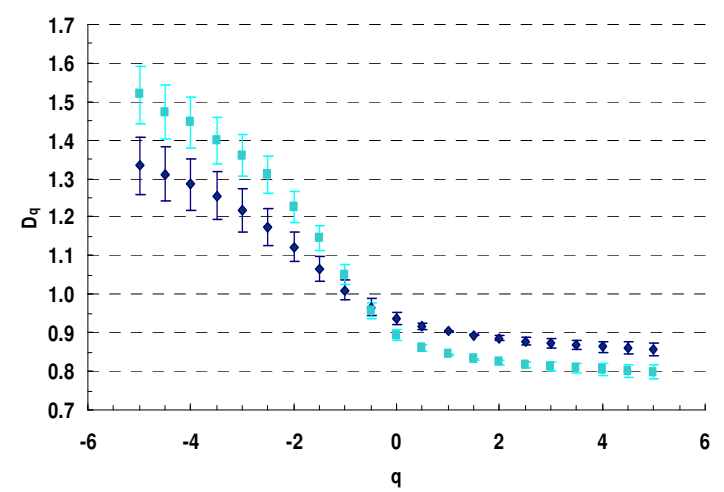

(c)

Fig. 3. Generalized dimensions $\left(D_{q}\right)$ from $q=-5$ to $q=+5$ for points of the regression line based on the bi-log plot of $\chi(a, q)$ to the range of areas from $512 \times 1024$ pixels to: (a) $8 \times 16$ pixels; (b) $16 \times 32$ pixels, (c) $32 \times 64$ pixels and (d) $64 \times 128$ pixels. Bars correspond to the standard errors of the estimation.

size. The next higher value, according to $2^{k}$, was $64 \times 128$ $(k=6)$ for both river basins (Table 1). The maximum box size corresponds to the biggest box we have analyzed, $512 \times 1024$ pixels. Confirmed by the configuration entropy and the attempt to maximize $R^{2}$ values and visual observations of the partition function, the box size range that should be chosen is from $64 \times 128$ till $512 \times 1024$ pixels for both river basins. The corresponding generalized dimensions based on the selected range of box sizes are shown in Fig. 3d. They all exhibit pronounced decreasing $D_{q}$ values with increasing $q$ and reveal a similar behavior. This problem has also been examined in detail by Saucier and Muller (1993, 1998, 1999), who proposed a systematic method to choose the scaling range for multifractal analysis. The advantage of their method is that a minimum and maximum box size is selected, however, in computational terms the MFA would become more complicated.

The standard errors of the $D_{q}$ estimates were lowest in the linear region (error bars in Fig. 3d), and the corresponding $R^{2}$ values were much higher (data not shown). This is not surprising if we realize that only three points are being used. In addition, the number of boxes of each size is very low, for size $64 \times 128$ pixels the number of boxes is 64 , for size $128 \times 256$ pixels the number of boxes is 16 , for size $256 \times 512$ pixels the number of boxes is 4 when analyzing an image of $512 \times 1024$ pixels.

Regardless that both river networks were fitted in a rectangle of $512 \times 1024$ pixels and the planes were subdivided with a box following this proportion $(1 \times 2)$, the effect of the drainage basins' shape is not totally eliminated, creating an underestimation of $\chi(a, q)$ values for larger boxes. However, the bias due to the shape of the drainage basins, is smaller than the bias associated with the limited resolution that affects the smaller boxes.

Detailed results for $D_{0}, D_{1}$ and $D_{2}$ can be found in Table 2, including the $q$ ranges where the mass exponent $\tau(q)$ exceeds the $R^{2}$ values higher than 0.95 . For values of $D_{0}$, the capacity dimensions are 0.89 for Tajo and 0.94 for Ebro, taking into account that the scaling has been based on area size and not on length. Even when a range of sizes is selected an overestimation of $D_{0}$ can result, achieving a Euclidian dimension (Dathe et al., 2006; Grau et al., 2006), where $D_{0}$ values are less than 1 . 
Table 2. Generalized dimensions $\left(D_{q}\right)$ for the first three positive moments $q$ for both river basins. Ranges of $\mathrm{q}$ where the slope of the partition function exceeds $R^{2} \geq 0.95$ are given, as well as the standard errors of the slope and the corresponding $R^{2}$.

\begin{tabular}{ccccccccccc}
\hline River & $-\mathrm{q}$ & $+\mathrm{q}$ & $\mathrm{D}_{0}$ & $\mathrm{R}^{2}$ & $\mathrm{D}_{1}$ & $\mathrm{R}^{2}$ & $\mathrm{D}_{2}$ & $\mathrm{R}^{2}$ & $\Delta\left(\mathrm{D}_{0}-\mathrm{D}_{5}\right)$ & $\Delta\left(\mathrm{D}_{-5}-\mathrm{D}_{5}\right)$ \\
\hline Tajo & -5.0 & 5 & $0.89 \pm 0.03$ & 1.00 & $0.84 \pm 0.00$ & 1.00 & $0.82 \pm 0.01$ & 0.99 & 0.096 & 0.721 \\
Ebro & -2.5 & 5 & $0.94 \pm 0.01$ & 1.00 & $0.90 \pm 0.00$ & 1.00 & $0.89 \pm 0.02$ & 0.99 & 0.081 & 0.477
\end{tabular}

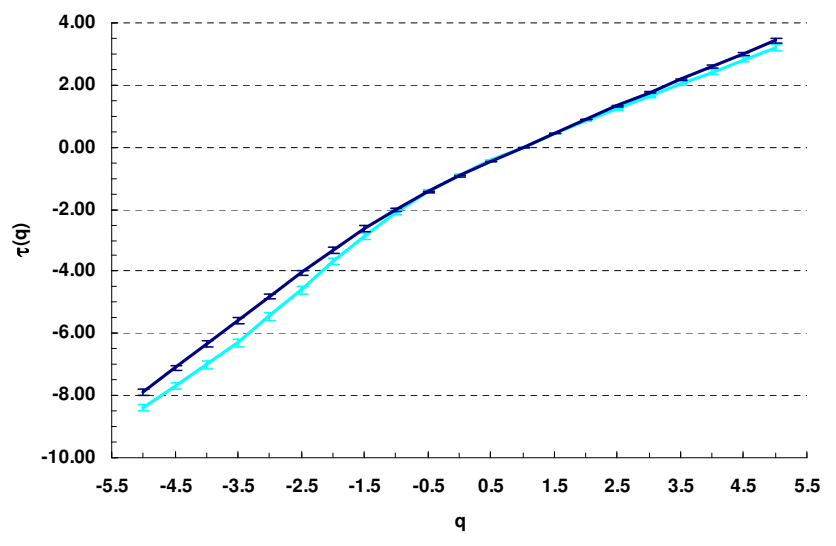

Fig. 4. The $q$ th mass exponent $(\tau(q))$ obtained with the gliding box method, from $q=-5$ to $q=+5$, using the range of areas from $512 \times 1024$ to $64 \times 128$ pixels for: (a) Tajo (light blue) and (b) Ebro (dark blue). Bars represent the standard errors of the estimation.

The main distinction between both river networks is the difference between $D_{-5}$ and $D_{5} ; \Delta\left(D_{-5}-D_{+5}\right)=0.721$ is found for Tajo and $\Delta\left(D_{-5}-D_{+5}\right)=0.477$ for Ebro is smaller. The differences in the generalized dimensions for $q=0,1,2$ can also be observed in Fig. 3d, where the lower part of the $D q$ curves $(q \geq 0)$ decreases more for Tajo than for the Ebro river network and it can be quantified by $\Delta\left(D_{-5}-D_{+5}\right)$ (Table 2).

Standard errors given in Table 2 are the standard errors of the slope obtained with linear regression. The smallest standard error which could be found is se $=0.00$ for $D_{1}$ of the Tajo and Ebro river network.

\subsection{Generalized dimensions using gliding box method}

The size range restriction found in the box counting method is avoided using the gliding box method. For both river networks, $<M(q, a)>$ was calculated and then a bi-log plot of $<M(q, a)>$ versus $\frac{a}{a_{\min }}$ was made, in order to estimate $\tau(q)$ (Fig. 4). All plots showed a linear relationship, as expected, for a large number of points, to calculate a linear regression in the range of box sizes selected before, and this is reflected in the low values of the standard errors of the estimations (Fig. 4, bars of each point). Both rivers show an almost identical $\tau(q)$ from $\mathrm{q}=-1$ till $\mathrm{q}=2$. In addition, both of them present $\tau(q=1)=0$, characterizing a conservative mea-

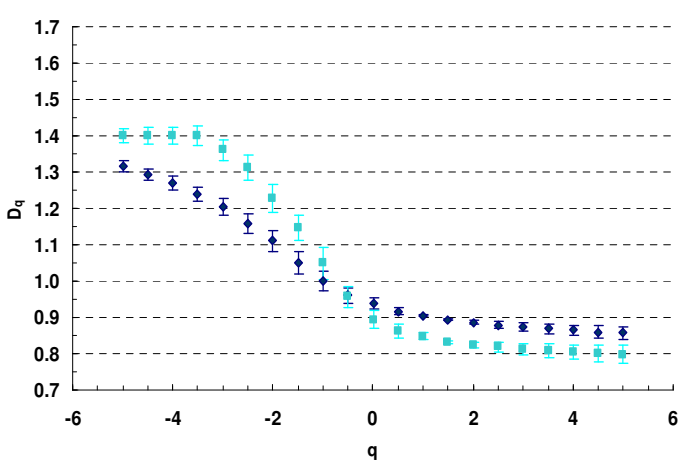

(a)

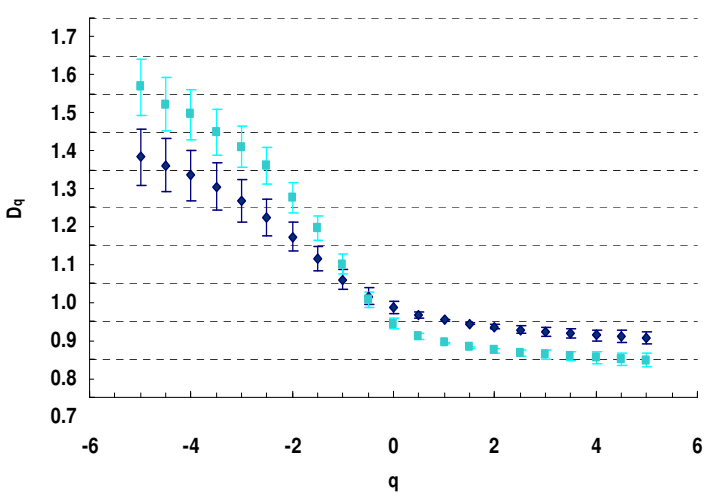

(b)

Fig. 5. Generalized dimensions $\left(D_{q}\right)$ from $q=-5$ to $q=+5$ based on the Tajo (light blue) and Ebro (dark blue) using the same box area ranges based on: (a) box-gliding; (b) box-counting. Bars represent the standard errors.

sure (Cheng, 1997b). For $q \leq-1$ a significant difference in the $\tau(q)$ curve between both river networks is observed.

Values of $D_{q}$ were calculated and a comparison between both methods for each river network was studied. In the gliding box method (Fig. 5a) both curves point out statistical differences for negative $q$ values, showing smaller standard errors than with the box counting method (Fig. 5b). Thanks to this reduction in the standard errors the comparison of $\Delta\left(D_{-5}-D_{+5}\right)$ and $D_{q}$ values for negative $q$ are statistically more significant. Another important advantage of the GB method is the reduction in the shape effect, due to the overlap of the boxes. This creates a higher number of boxes including the drainage basin area. 
In observing the difference between $D_{q}$ for both methods there is always a reduction in the GB method than in the BC method; however, the standard errors are smaller with the first method. In the case of positive $q$ values both methods gave the same results, which are in agreement with those found by Grau et al. (2006) for pore systems.

\section{Conclusions}

Generalized dimensions $D_{q}$ have been obtained for the Tajo and Ebro river networks by applying the box counting method using a different range of box areas $(a)$. Depending on the range selected $D_{q}$ estimation leads to clearly distinct values with high standard errors. This study has illustrated how crucial the range of box sizes chosen is in determining $D_{q}$ values, especially for negative $q$ moments. This is even more important when binary images (build up with 0 and 1 element) are analyzed and only a few pixels can cause great changes in the partition function when $q<0$.

We have applied the configuration entropy, following the work of Tarquis et al. (2006) and Dathe et al. (2006), to select the minimum area that should be used, in order to obtain good results in the $D_{q}$ estimations but realizing that the number of boxes measured and the number of points for the regression analysis was low.

Using the same area range, the $D_{q}$ values have been calculated by applying the gliding box method. The results obtained by the "box-counting" and "gliding-box" methods for multifractal modeling of river networks show that the "gliding-box" provides more consistent results due to the creation of a greater number of large size boxes in comparison with the "box-counting" method, mainly when $q<0$, obtaining a higher number of points for the regression analysis and reducing the bias associated with the shape of the drainage basins.

In terms of modeling, it is important to characterize the multiscale heterogeneity of river networks in a useful way, but the application of fractal/multifractal analyses should be done carefully to avoid problems in interpretation and model building.

Acknowledgements. The authors would like to express their gratitude for the work and patient of the two anonymous reviewers, as well as Quiming Cheng and J. M. Gascó, who have improved the manuscript. This work has been partially supported by CM-UPM under Project (R05/11261) and Project (CCG06-UPM/AGR22). We thank to Agencia Catalana del Agua (Generalitat de Catalunya) for the research contract with Fundacin Premio Arce (E.T.S.I. Agrónomos, Universidad Politcnica de Madrid).

Edited by: Q. Cheng

Reviewed by: two anonymous referees

\section{References}

Ahammer, H., DeVaney, T. T. J., and Tritthart, H. A.: How much resolution is enough? Influence of downscaling the pixel resolution of digital images on the generalized dimensions, Physica D, 181, 147-156, 2003.

Aharony, A.: Measuring Multifractals, Physica D, 38, 1-4, 1989.

Aharony, A.: Multifractals in physics: Successes, dangers and challenges, Physica A, 168, 479-489, 1990.

Allain, C. and Cloitre, M.: Characterizing the lacunarity of random and deterministic fractal sets, Phys. Rev. A, 44, 3552-3558, 1991.

Andraud, C., Beghdadi, A., Haslund, E., Hilfer, R., Lafait, J., and Virgin, B.: Local entropy characterization of correlated random microstructures, Physica A, 235, 307-318, 1997.

Andraud, C., Beghdadi, A., and Lafait, J.: Entropic analysis of random morphologies, Physica A, 207, 208-217, 1994.

Barnsley, M. F., Devaney, R. L., Mandelbrot, B. B., Peitgen, H. O., Saupe, D., and Voss, R. F.: The Science of Fractal Images, edited by: Peitgen, H. O. and Saupe, D., Springer-Verlag, New York, 1988.

Beauvais, A. A. and Montgomery, D. R.: Are channel networks statistically self-similar?, Geology, 25, 1063-1066, 1997.

Beghdadi, A., Andraud, C., Lafait, J., Peiro, J., and Perreau, M.: Entropic and multifractal analysis of disordered morphologies, Fractals 3, 671-679, 1993.

Bird, N., Díaz, M. C., Saa, A., and Tarquis, A. M.: Fractal and Multifractal Analysis of Pore-Scale Images of Soil, J. Hydrol., 322, 211-219, 2006.

Block, A., von Bloh, W., and Schellnhuber, H. J.: Efficient boxcounting determination of generalized fractal dimensions, Phys. Rev. A, 42, 1869-1874, 1990.

Buczhowski, S., Hildgen, P., and Cartilier, L.: Measurements of fractal dimension by box-counting: a critical analysis of data scatter, Physica A, 252, 23-34, 1998.

Cheng, Q.: Discrete multifractals, Math. Geol., 29(2), 245-266, 1997a.

Cheng, Q.: Multifractal modeling and lacunarity analysis, Math. Geol., 29(7), 919-932, 1997b.

Cheng, Q. and Agerberg, F. P.: Comparison between two types of multifractal modeling, Math. Geol., 28(8), 1001-1015, 1996.

Cheng, Q.: Multifractality and spatial statistics, Comp. Geosci., 255, 949-961, 1999.

Chhabra, A. B., Menevau, C., Jensen, R. V., and Sreenivasan, K. R.: Direct determination of the $f(\alpha)$ singularity spectrum and its application to fully developed turbulence, Phys. Rev. A, 40, 5284-5294, 1989.

Claps, P. and Oliveto, G.: Reexamining the determination of the fractal dimension of river networks, Water Resour. Res., 32, 3123-3135, 1996.

Coniglio, A. and Zannetti, M.: Multiscaling and multifractality, Phys. D, 38, 37-40, 1989.

Cudennec, C., Fouad, Y., Sumarjo Gatot, I., and Duchesne, J.: A geomorphological explanation of the unit hydrograph concept, Hydrol. Processes, 18(4), 603-621, 2004.

Dathe, A., Tarquis, A. M., and Perrier, E.: Multifractal analysis of the pore- and solid-phases in binary two-dimensional images of natural porous structures, Geoderma, 134, 318-326, 2006.

De Bartolo, S. G., Primavera, L., Gaudio, R., D’Ippolito, A., and Veltri, M.: Fixed-mass multifractal analysis of river networks 
and braided channels, Phys. Rev. E, 74, 026101(8), 2006.

De Bartolo, S. G., Veltri, M., and Primavera, L.: Estimated generalized dimensions of river networks, J. Hydrol., 322, 181-191, 2006.

De Bartolo, S. G., Gaudio, R., and Gabriele, S.: Multifractal analysis of river networks: Sandbox approach, Water Resour. Res., 40, W02201, doi:10.1029/2003WR002760, 2004.

De Bartolo, S. G., Gabriele, S., and Gaudio, R.: Multifractal behaviour of river networks, Hydrol. Earth Syst. Sci., 4, 105-112, 2000 ,

http://www.hydrol-earth-syst-sci.net/4/105/2000/.

Evertsz, C. J. G. and Mandelbrot, B. B.: Multifractal Measures, in: Chaos and Fractals, edited by: Peitgen, H., Juergens, H., and Saupe, D., Springer, Berlin, pp 921-953, 1992.

Feder, J.: Fractals, Plenum Press, New York, 1989.

Feeny, B. F.: Fast multifractal analysis by recursive box covering, Int. J. Bifurcat. Chaos, 10, 2277-2287, 2000.

Fiorentino, M., Oliveto, G., and Rossi, A.: Alcuni aspetti del controllo energetico ed idrologico sulla geometria delle reti e delle sezioni fluviali, Parte prima: controllo idrologico, Atti del XXVIII Convegno di Idraulica e Costruzioni Idrauliche, Potenza, Editoriale Bios Cosenza, 1, 71-78, 2002.

Gascó, G., Saa, A., Díaz, M. C., and Tarquis, A. M.: Multifractal Scaling of River Networks: A Case Study of Tajo and Ebro Rivers, Geophys. Res. Abstr., 6, 07016, 2004.

Gaudio, R., De Bartolo, S. G., Primavera, L., Gabrielle, S., and Veltri, M.: Lithologic control on the multifractal spectrum of river networks, J. Hydrol., 327, 365-375, 2006.

Grassberger, P. and Procaccia, I.: Characterization of strange attractors, Phys. Rev. Lett., 50, 346-349, 1983.

Grassberger, P.: Generalized dimensions of strange attractors, Phys. Lett. A, 97, 227-230, 1983.

Grau, J., Médez, V., Tarquis, A. M., Saa, A., and Díaz, M. C.: Comparison of gliding box and box-counting methods in soil image analysis, Geoderma, 134, 349-359, 2006.

Gupta, V. K., Mesa, O. J., and Dawdy, D. R.: Multiscaling theory of flood peaks: quantile analysis, Water Resour. Res., 30, 34053421, 1994.

Hasley, T. C., Jensen, M. H., Kadanoff, L. P., Procaccia, I., and Shraiman, B. I.: Fractal Measures and their Singularities - the Characterization of Strange Sets, Phys. Rev. A, 33(2), 11411151, 1986.

Hentschel, H. G. R. and Procaccia, I.: The infinite number of generalized dimensions of fractals and strange attractors, Physica D, 8, 435-444, 1983.

Hjelmfelt, A. T.: Fractals and the river-length catchment-area ratio, Water Resour. Bull., 24, 455-459, 1988.

Horton, R. E.: Erosional development of streams and their drainage basins: Hydrophysical approach to quantitative geomorphology, Geol. Soc. Am. Bull., 56, 275-370, 1945.

Horton, R. E.: Drainage basin characteristics, Transactions of the American Geophysical Union (AGU), 13, 350-370, 1932.

Ijjasz-Vasquez, E. J., Rodríguez-Iturbe, I., and Bras, R. L.: On the multifractal characterization of river basins, Geomorphology, 5, 297-310, 1992.

Ijjász-Vasquez, E. J., Brass, R. L., Rodriguez Iturbe, I., Rigon, R., and Rinaldo, A.: Are river basins optimal channel networks?, Adv. Water Resour., 16, 69-79, 1993.

La Barbera, P. and Rosso, R.: On the fractal dimension of stream networks, Water Resour. Res., 25, 735-741, 1989.

Lavallée, D., Lovejoy, S., Schertzer, D., and Ladoy, P.: Non-linear variability of landscape topography: multifractal analysis and simulation, in: Fractals in Geography, edited by: Lam, N. S. and De Cola, L., Prentice Hall, Englewood Cliffs, NJ, 158-192, 1993.

Liebovitch, L. S. and Toth, T.: A fast algorithm to determine fractal dimensions by box-counting, Phys. Lett. A, 141, 386-390, 1989.

Mach, J., Mas, F., and Sagués, F.: Two representations in multifractal analysis, J. Phys. A Math. Gen., 28, 5607-5622, 1995.

Mandelbrot, B. B.: Fractals: form, chance and dimension, Freeman, S. Francisco, 1977.

Mandelbrot, B. B.: The fractal geometry of nature, Freeman, S. Francisco, 1982.

Maritan, A., Rinaldo, A., Rigon, R., Giacometti, A., and RodriguezIturbe, R.: Scaling laws for river networks, Phys. Rev. E, 53, 1510-1515, 1996.

Meakin, P.: Fractals, scaling and growth far from equilibrium, Cambridge University Press, Cambridge (UK), 1998.

Mesa, O. J. and Gupta, V. K.: On the main channel length-area relationships for channel networks, Water Resour. Res., 23, 21192122, 1987.

Oiwa, N. N. and Fiedler-Ferrara, N.: A moving-box algorithm to estimate generalized dimensions and $\mathrm{f}(\alpha)$ spectrum, Physica D, 124, 210-224, 1998.

Pardé, M.: Fleuves et Rivières, Colin, Paris, 224 pp, 1955.

Peckham, S.: New results for self-similar trees with application to river networks, Water Resour. Res., 31, 1023-1029, 1995.

Perrier, E., Tarquis, A. M., and Dathe, A.: A Program for Fractal and Multifractal Analysis of Two-Dimensional Binary Images. Computer Algorithms versus Mathematical Theory, Geoderma, 134, 284-294, 2006.

Plotnick, R. E., Gardner, R. H., Hargrove, W. W., Prestegaard, K., and Perlmutter, M.: Lacunarity analysis: A general technique for the analysis of spatial patterns, Phys. Rev. E, 53(5), 5461-5468, 1996.

Rényi, A.: On a new axiomatic theory of probability, Acta Mathematica Hungarian, VI(3-4), 285-335, 1955.

Rigon, R., Rinaldo, A., Rodriguez-Iturbe, I., Bras, R. L., and Vasquez, E. J.: Optimal channel networks: a frameworkfor the study of river basin morphology, Water Resour. Res., 29, 16351646, 1993.

Rinaldo, A. and Rodríguez-Iturbe, I.: Channel Networks, Ann. Rev. Earth Planet. Sci., 26, 289-327, 1998.

Rinaldo, A., Marani, A., and Rigon, R.: Geomorphological dispersion, Water Resour. Res., 27, 513-525, 1991.

Rinaldo, A., Rodriguez-Iturbe, I., Rigon, R., Vasquez, E. J., and Bras, R. L.: Self-organized fractal river networks, Phys. Rev. Lett., 70, 822-825, 1993.

Rinaldo, A., Rodriguez-Iturbe, I. , Rigon, R., Bras, R. L., and Vasquez, E. J.: Minimum energy and fractal structures of drainage networks, Water Resour. Res., 28, 2183-2195, 1992.

Rodriguez Iturbe, I., Marani, M., Rigon, R., and Rinaldo, A.: SelfOrganized river basin landscape: Fractal and multifractal characteristics, Water Resour. Res., 30, 3531-3539, 1994.

Rodriguez-Iturbe, I. and Rinaldo, A.: Fractal river basins. Change and self-organization, Cambridge University Press, Cambridge, UK, 1997.

Rodriguez-Iturbe, I. and D’Odorico, P.: Configuration Entropy of 
fractal landscapes, Geophys. Lett., 25, 1015-1018, 1998.

Saucier, A. and Muller, J.: Remarks on some properties of multifractals, Physica A, 199, 350-358, 1993.

Saucier, A. and Muller, J.: Multifractal approach to textural analysis, in: Fractals and beyond, complexities in the sciences, edited by: Novak M. M., World Scientific Publishing Co., London, pp. 161-171, 1998.

Saucier, A. and Muller, J.: Textural analysis of disordered materials with multifractals, Physica A, 267, 221-238, 1999.

Sundborg, A. and Jansson, M.: Hydrology of rivers and river regimes, in: Temperate Palaeohydrology, edited by: Starkel, L., Gregory, K. J., and Thornes, J. B., John Wiley \& Sons, Chichester, England, 548 pp, 1991.

Tarboton, D. G., Bras, R. L., and Rodriguez-Iturbe, I.: The fractal nature of river networks, Water Resour. Res., 24, 1317-1322, 1990.

Tarquis, A. M., Giménez, D., Saa, A., Díaz, M. C., and Gascó, J. M.: Scaling and Multiscaling of Soil Pore Systems Determined by Image Analysis, in: Scaling Methods in Soil Physics, edited by: Pachepsky, Y., Radcliffe, D. E., and Selim, H. M., CRC Press, 434 pages, 2003.

Tarquis, A. M., Losada, J. C., Benito, R., and Borondo, F.: Multifractal analysis of the Tori destruction in a molecular Hamiltonian System, Phys. Rev. E., 65, 0126213(9), 2001.
Tarquis, A. M., McInnes, K., Keys, J., Saa, A., García, M. R., and Díaz, M. C.: Multiscaling Analysis in a Structured Clay Soil Using 2D Images, J. Hydrol., 322, 236-246, 2006.

Tel, T., Fulop, A., and Vicsek, T.: Determination of fractal dimensions for geometrical multifractals, Phys. A, 159, 155-166, 1989.

Tel, T. and Vicsek, T.: Geometrical multifractality of growing structures, J. Phys. A. General, 20, L835-L840, 1987.

Veltri, M., Veltri, P., and Maíolok, M.: On the fractal description of natural channel networks, J. Hydrol., 187, 137-144, 1996.

Veneziano, D., Moglen, G. E., and Bras, R. L.: Multifractal analysis: pitfalls of standard procedures and alternatives, Phys. Rev. E, 52, 1387-1398, 1995.

Veneziano, D. and Niemann, J. D.: Self-similarity and multifractality of fluvial erosion topography. 1. Mathematical conditions and physical origin, Water Resour. Res., 36, 1923-1936, 2000a.

Veneziano, D. and Niemann, J. D.: Self-similarity and multifractality of fluvial erosion topography. 2. Scaling properties, Water Resour. Res., 36, 1937-1952, 2000b.

Vicsek, T.: Mass multifractals, Physica A, 168, 490-497, 1990.

Vicsek, T., Family, F., and Meakin, P.: Multifractal geometry of diffusion-limited aggregates, Europhys. Lett., 12, 217-222, 1990.

Yamaguti, M. and Prado, C.: Smart covering for a box-counting algorithm, Phys. Rev. E, 55, 7726-7732, 1997. 